\title{
Probabilistic Estimation of Network Size and Diameter
}

\author{
Jorge C. S. Cardoso \\ E.Artes / CITAR \\ Universidade Católica Portuguesa (UCP) \\ Porto, Portugal \\ Email: jorgecardoso@ieee.org
}

\author{
Carlos Baquero and Paulo Sérgio Almeida \\ $\mathrm{DI} / \mathrm{CCTC}$ \\ Universidade do Minho \\ Braga, Portugal \\ Email: \{cbm,psa\}@di.uminho.pt
}

\begin{abstract}
Determining the size of a network and its diameter are important functions in distributed systems, as there are a number of algorithms which rely on such parameters, or at least on estimates of those values.

The Extrema Propagation technique allows the estimation of the size of a network in a fast, distributed and fault tolerant manner. The technique was previously studied in a simulation setting where rounds advance synchronously and where there is no message loss.

This work presents two main contributions. The first, is the study of the Extrema Propagation technique under asynchronous rounds and integrated in the Network Friendly Epidemic Multicast (NeEM) framework. The second, is the evaluation of a diameter estimation technique associated with the Extrema Propagation. This study also presents a small enhancement to the Extrema Propagation in terms of communication cost and points out some other possible enhancements.

Results show that there is a clear trade-off between time and communication that must be considered when configuring the protocol-a faster convergence time implies a higher communication cost. Results also show that its possible to reduce the total communication cost by more than $18 \%$ using a simple approach. The diameter estimation technique is shown to have a relative error of less than $10 \%$ even when using a small sample of nodes.
\end{abstract}

Keywords-Aggregation; Network Size Estimation; Network Diameter Estimation; Probabilistic Estimation;

\section{INTRODUCTION}

Determining the size of a network is an important function in a distributed system. There are a number of algorithms which rely on an estimate of the network size, or that would at least benefit from such an estimate, for example: distributed hash tables can take advantage of having an estimate of the network size to adjust the size of the routing table that each node keeps; gossip-based protocols [1], [2] can use an estimate of the network size to better adjust the gossiping fanout parameter;

Although important, determining the size of a network, in a distributed manner, is not trivial. Algorithms that do this should be fast, to cope with high churn; fault-tolerant, to cope with link and node failures; and use a small number of messages, in order not to impose a high overhead in network bandwidth.

Another important network parameter is its diameter-the maximum shortest-path length between any two pairs of nodes. Knowing the diameter, or at least having an estimate of its value is important to configure, for example, the time-to-live field on many protocols.
In a previous work, [3], [4] introduced the Extrema Propagation technique, which allows the estimation of the size of a network. This technique is fast because it produces estimates in a number of steps close to the theoretical minimum; completely distributed, because every node determines the estimate by itself; does not require global identifiers; and tolerates message loss. Also, it is possible to adapt it in order to have it produce an estimate of the network diameter.

However, the Extrema Propagation technique was originally studied in a simulation setting where rounds advance synchronously and where there is no message loss. In a real scenario, networks and nodes are often not synchronous, some nodes may fail, links have different latencies and messages may be lost in transit. The purpose of this work is to:

- study the Extrema Propagation technique under asynchronous rounds;

- extend the technique in order to have it produce an estimate of the network diameter;

- evaluate an optimization to the original message size of the Extrema Propagation technique.

In order to study the technique in a more realistic setting, we adapted and integrated it in the Network Friendly Epidemic Multicast [5] (NeEM) framework ${ }^{1}$ since NeEM could also benefit from the estimates produced by Extrema.

This paper is organized as follows: Section II describes some algorithms that perform data aggregation across a network and compares them to the algorithm used in this paper. Section III describes the NeEM software that was modified and used in this work. Section IV gives an overview of the Extrema Propagation technique for estimating the size of a network and how it can be adapted to estimate the network's diameter. Section $\mathrm{V}$ describes the general experimental procedure and the various experiments performed. Section VI presents the results from the experiments and, finnaly, Section VII concludes.

\section{RELATED WORK}

There are numerous algorithms for estimating the size of a network or, more generally, for performing data aggregation across a network.

Some algorithms, more directly related to this study are described next.

\footnotetext{
${ }^{1} \mathrm{NeEM}$ is a software framework for group communication based on gossip protocols.
} 
The work in [6] presents two methods for estimating the size of a network: the Hops Sampling and the Interval Density methods.

In the Hops Sampling method, an initiator node starts the process by sending a message to gossipTo nodes. The message contains a hop count that is incremented prior to being sent. Messages sent from a node $p$ are only sent to nodes from which $p$ has not received any message. After gossipResults rounds have elapsed the initiator collects a sample of hop counts from gossipSample other nodes and uses the average of the hop counts as an estimate of $\log (N)$. However, contrary to the Extrema technique, this methods requires that each node is able to maintain a membership list chosen uniformly at random from the system. Also, only the initiator node will have an estimate of the size.

In the Interval Density method, the process identifiers are hashed and mapped to a point in the interval $[0,1]$. The initiator node then calculates the number of processes, $X$, in an interval $I<1$. The estimate of the network size is then calculated as $X / I$. In order to collect the samples (identifiers) the authors propose piggybacking messages from a membership maintenance protocol with information about the interval used in the estimates. In this method, the difficulty is in defining the interval $I$, since it depends on the network size $N$.

Push-sum [7] introduces a different approach for computing sums and averages and can provide high precision estimates after a sufficient number of rounds. Each node keeps two variables: $s$ and $w$ initialized to $x_{i}$ (the contribution of node $i$ to the sum) and 1 (the initial weight of this contribution), respectively. In each round, nodes select another node at random and send $\frac{s}{2}$ and $\frac{w}{2}$ to that node (and keep $\frac{s}{2}$ and $\frac{w}{2}$ to themselves). Each pair of $s$ and $w$ received by a node is added to the current ones. In each round, the estimate of the average is given locally by $\frac{s}{w}$. After a number of rounds all nodes converge to the same result, the global average.

The push-pull algorithm [8] introduces a similar approach but in this case information is exchanged in a symmetric manner. A node $A$ randomly selects another node $B$ to exchange its value $v_{a}$ and sends him a Push message with $v_{a}$. Upon receipt of a Push message, node B will send a Pull message with its own value $v_{b}$ to the node A. After receiving a Push or a Pull message both nodes will update their value $v$ :

$$
v=\frac{v_{a}+v_{b}}{2}
$$

This technique (as well as the one by [7]) is sensitive to message loss. Both algorithms require that the total system "mass" is kept constant. A lost message will break this requirement. In Extrema, message loss does not affect in the long run the result of the algorithm since "each message is made obsolete by subsequent ones: if a message from A to B containing vector $x$ is lost, a subsequent message will have content $y$, where $y \leq x$ (in pointwise order)" [4].

\section{NEEM}

NeEM - Network Friendly Epidemic Multicast [9] - is an epidemic multicast protocol which relies on connectionoriented transport connections (TCP/IP) in order to take advantage of the built-in end-to-end congestion control.

When gossiping, NeEM combines different strategies. If the message to be gossiped is small (smaller than a predefined value), then it is always pushed until its time-to-live (TTL) has expired. If the message is large, then it is pushed during PushTTL rounds, and then advertised until TTL has expired. Both TTL and PushTTL are preconfigured.

NeEM has been implemented in $\mathrm{Java}^{2}$ and its main components are:

Multicast channel Provides applications with an interface for joining and leaving the network and for receiving and multicasting messages. In order to join the network, a peer needs to know, at least, another peer already in the network which will act as the entry point. A peer is identified by a randomly generated universally unique identifier (UUID).

Gossip Layer Takes care of gossiping messages using the strategies described earlier. Keeps a cache of messages so that it can respond to pulling by peers. It also keeps a list of known message advertisers in order to pull advertised messages. Messages are identified by a randomly generated UUID. When gossiping, it randomly selects Fanout peers from the partial membership kept by the overlay layer.

Overlay Layer Manages the overlay network and a partial membership list. The overlay tries to maintain a fixed number of peers in the local membership list (in NeEM's implementation this is also called Fanout but is different from the gossip Fanout). Membership is dynamic as the overlay will shuffle peers periodically, by randomly selecting two peers from the current list and informing them of one another. Those peers will then randomly decide if they add the other one to the current list, eventually purging another.

Transport Layer The transport layer manages TCP connections between peers, maintaining queues of messages per connection.

NeEM's structure can be seen as layer stack as depicted in Figure 1.

\begin{tabular}{|c|}
\hline Application \\
\hline Multicast Channel \\
\hline Gossip \\
\hline Overlay \\
\hline Transport \\
\hline
\end{tabular}

Fig. 1. NeEM's layer stack.

NeEM is implemented in a way that allows easy integration of other protocols. The transport layer allows different handlers to be registered and will demultiplex messages based on a logical port and deliver them to the appropriate handler.

\footnotetext{
${ }^{2}$ See the project's sourceforge web page: http://neem.sourceforge.net/index.
} html. 
Peers join a NeEM group by contacting another known peer, however, even if all peers join through the same known peer, the overlay topology will always tend to a random network, since NeEM shuffles peers periodically by performing a random walk.

\section{Extrema Propagation}

The Extrema Propagation technique is a probabilistic data aggregation technique which works as follows.

"[...] if we generate a random real number in each node using a known probability distribution (e.g. Gaussian or exponential), and aggregate across all nodes using the minimum function, the resulting value has a new distribution which depends on the number of nodes. The basic idea is then to generate a vector of random numbers at each node, aggregate each component across the network using the pointwise minimum, and then use the resulting vector as a sample from which to infer the number of nodes (by a maximum likelihood estimator)." [4]

Basically, each node generates a vector of $K$ exponentially distributed random numbers (Minimums vector in the rest of this document) and sends it to its neighbours. Each node aggregates and resends vectors from neighbours using the pointwise minimum - an idempotent operation. After convergence (determined by each node, after a predefined number of rounds have passed without changing the Minimums vector) the resulting vector-with the $K$ global minimums - can be used to estimate the number of nodes in the network.

The technique's focus is on speed not on accuracy, since aiming at very low errors would need very large vectors. For example, an error of $1 \%$ with $95 \%$ confidence would require

$$
K=2+\left(\frac{1.96}{\text { error }}\right)^{2}=2+\left(\frac{1.96}{0.01}\right)^{2}=38418 .
$$

A $10 \%$ error, however, only requires $K=387$.

In order to optimize the message's size, the technique encodes the values using only the exponent of its representation (mantissa, exponent). Furthermore, for most cases only 5 bits need to be used to represent the exponent. This means that a vector with $K=387$ needs only

$$
5 \times 387 \text { bits }=242 \text { bytes } .
$$

Convergence is determined by a predefined number of rounds without changes (no news rounds, $T$ ) occurring in the Minimums vector. The minimum number of no news rounds to wait depends on the network topology and size, but a safe value can be used to accommodate, at least, a wide range of expected network sizes.

The technique was studied by simulating runs (with synchronous rounds) for several values of $K$ and for different types of networks (geometric 2D, random and preferential attachment networks) and sizes (100, 1000 and 10000 nodes). For each run, the average and maximum number of rounds needed to converge where recorded, as well as the maximum number of rounds with no news.
Results show that, for random and preferential attachment networks, $T=5$ is sufficient when $K=10$ or $K=100$ and $T=4$ is sufficient when $K=1000$. For geometric $2 \mathrm{D}$ networks, $K$ should be 100 or 1000 since $K=10$ would lead to a large overhead of the no news rounds over the average number of rounds needed to converge. For $K=100$ a $T=19$ would suffice and for $K=1000, T=11$. These are all conservative values to ensure that all nodes have converged.

The Extrema Propagation technique is resilient to message failures: if a message (with vector $x$ ) from node $A$ to node $B$ is lost, in the next round, it will be superseded by another message (vector $x^{\prime}$ ) with $x^{\prime} \leq x$.

The synchronous version of the basic algorithm, though, does not cope with message loss since nodes are made to wait for all neighbours. A single message loss would deadlock the system. It is easy to adapt the algorithm and cope with message loss. As suggested in [4], the algorithm can be modified to, instead of waiting for every neighbour, forever:

- Wait for all neighbours, until a timeout occurs (ONLY_TIMEOUT strategy).

- Wait for all neighbours minus $F$ (ONLY_F strategy).

- Wait for all neighbours, until a timeout and then wait for all minus $F$ (TIMEOUT_PLUS_F strategy).

For sake of completeness, there is one more variant that can be used:

- Wait for all neighbours minus $F$, until a timeout occurs (F_PLUS_TIMEOUT) strategy.

These variants, besides allowing to cope with message failures, make the algorithm more robust in face of slow links. In the original algorithm, although not fatal, a single slow link would slow down the entire system. By introducing timeouts and the possibility of not waiting for every neighbour, a slow link can be regarded as a message failure. This means that the algorithm may not wait for nodes behind slow links, thus not slowing the entire round. Messages sent on slow links will arrive and be accounted for in the following rounds.

In Section V, one discusses experimental results that can guide the proper configuration of the timeout and $F$ in practical asynchronous settings.

\section{A. Diameter Estimation}

The Extrema Propagation technique can be augmented to also give an estimate of the network diameter: for each entry in the Minimums vector of the Extrema technique, one adds a corresponding entry in a new vector Hops. The Hops vector is initialized at every node with zeroes. Every time that a node updates the Minimums vector because it has received a smaller value from one of its neighbours, it also updates the corresponding entry in the Hops vector with the value received from that neighbour. The Hops vector is also updated when a node receives a smaller entry in the Hops vector for an equal value in the Minimums vector (meaning that a shorter route to that minimum was found). A copy of the Hops vector, with values increased by 1 , is sent along with the Minimums vector, to every neighbour, in each round. 
After convergence, in each node $\max$ (Hops) is an estimate of its eccentricity and the network diameter that can be determined across the network by aggregating a maximum of these eccentricities. In particular, if one of the minimums was generated in a periphery node the final diameter estimate will be exact.

\section{B. Message Size Optimization}

The standard message in the Extrema Propagation is a message with $K$ values, 5 bits each, corresponding to the minimums vector that each node stores. This vector is updated when one (or several) new minimums is received, but is sent in every round even if it is equal to the last vector sent. Also, the same vector is sent to every neighbour, regardless of what that neighbour already "knows".

This means that potentially old information is sent in each message. A simple way to mitigate this inefficiency is to store the last vector that each neighbour sent and use it as a way to determine what needs to be sent to that neighbour in the next round. This way, messages are differentiated by neighbour. A message only needs to contain values that are smaller than the ones in the current copy of that neighbours' vector.

Instead of sending $K * 5$ bits, each node can send only $N$ pairs of (index, value) entries - the number of values smaller than the ones in the current copy of that neighbour's vector. In order to correctly interpret the message, the receiving node needs to know also how many pairs were sent. This message will only save bandwidth if the number of pairs (index, value) is sufficiently small. If not, the standard format should be used instead.

Optimizing the message size in this way means trading off bandwidth usage for memory usage since each node now has to keep the last vectors received from each neighbour.

The final message structure uses one of two types:

\begin{tabular}{|l|r|}
\hline Type 0 & 1 bit \\
\hline Number of pairs $(N)$ & $\operatorname{ceil}\left(\log _{2}(K)\right)$ bit \\
\hline (Index, Value) pairs & $\left(\right.$ ceil $\left.\left(\log _{2}(K)\right)+5\right) * N$ bit \\
\hline
\end{tabular}

\begin{tabular}{|l|r|}
\hline Type 1 & 1 bit \\
\hline Minimums vector & $K * 5$ bit \\
\hline
\end{tabular}

In order for Type 0 to be used it must save bandwidth, which means that:

$$
\operatorname{ceil}\left(\log _{2}(K)\right)+\left(\operatorname{ceil}\left(\log _{2}(K)\right)+5\right) * N \leq K * 5
$$

This results in:

$$
\begin{gathered}
N \leq \frac{K * 5-\operatorname{ceil}\left(\log _{2}(K)\right)}{\operatorname{ceil}\left(\log _{2}(K)\right)+5} \\
\text { V. EXPERIMENTS }
\end{gathered}
$$

\section{A. Setup}

The evaluation of the integration of the Extrema Propagation technique in NeEM was done using Modelnet [10] as the simulation platform.
The following is a description of the general simulation setup and procedures that were used.

The experiment network consisted of two computers one emulator and one application host with the following characteristics:

Emulator:

- Pentium $4 \mathrm{CPU}$ at $3.40 \mathrm{GHz}$

- 2 Gb RAM

- 3Com Etherlink XL 10/100 PCI TX NIC

Application host:

- Pentium $4 \mathrm{CPU}$ at $3.40 \mathrm{GHz}$

- $1.5 \mathrm{~Gb}$ RAM

- Broadcom NetXtreme Gigabit Ethernet

Modelnet's graph file was created with the following command:

inet $-\mathrm{n} 3037$ inet $2 \mathrm{xml}-1$-p 800 ।

among 50 stubs min-client-stub $100010 \backslash$

max-client-stub 1000500

Which creates a network of 3037 nodes (the minimum for inet) and 800 clients $^{3}$ attached among 50 stubs $^{4}$. The command also specifies the bandwidth of the client-stub link to be $1000 \mathrm{kbps}$, latency between $[1,50]$ milliseconds and no packet drops. All the other link types have default parameters, except for latency which is inferred from the node distance.

The experiments were controlled by a set of shell scripts that invoked specific JMX (Java Management Extensions) functions on a Java class that represented the experiment. This class was responsible for loading all instances of the modified multicast channel, connecting them, starting the Extrema protocol and wait for it to finish.

The process of running an experiment consisted of:

1) Loading the main experiments class;

2) Adding $\mathrm{N}$ nodes by randomly picking $\mathrm{N}$ virtual node addresses from the set assigned to the machine;

3) Configuring each node according to the experiment settings;

4) Randomly connecting nodes among themselves;

5) Letting overlay network settle for a few seconds;

6) Starting the extrema protocol on one randomly chosen node;

7) Waiting until a preconfigured number of rounds had elapsed;

8) Stopping the experiment;

9) Saving the $\log$ files.

The log files recorded the following:

- Message send timestamp;

- Message receive timestamp;

- After each round: round number, current Minimums vector, current Hops vector, timestamp, number of total sent messages.

${ }^{3} \mathrm{~A}$ client node in Modelnet's terminology is an edge node in the virtual network corresponding to a computer attached to the wide area internet [11, p. 10].

\footnotetext{
${ }^{4} \mathrm{~A}$ stub is a gateway for client nodes to access the network [11, p. 10].
} 
Running several instances of the protocol on the same machine has two potential bottlenecks: the CPU and the network interface.

In the case of our experiments, the communication load of each node was fairly small so bandwidth was not a problem. On the other hand, running several threads of the protocol in the Java virtual machine turned out to be very CPU intensive.

The latency between nodes, for an increasing number of nodes and for different configurations, was measured and the results showed that, for some configurations and number of nodes, the latency between nodes increased beyond that imposed by Modelnet (and the message processing overhead at the machine).

This restriction led to the use the following maximum values in most of the experiments: $K=10$, MaximumNumberOf Nodes $=75$ and OverlayFanout $=$ 10.

In all experiments that used this modified NeEM implementation, the network topology used was the one that emerges through NeEM's automatic shuffling of peers: an approximately random network.

\section{B. Timeout and F}

In order to determine the best combination of Timeout and $F$ to use in the NeEM Extrema protocol, several combinations were tested.

The base values for the tested parameters were Timeout $=$ $200, F=0.8$ (in the rest of this document, $F$ is the fraction of neighbours to wait for) and the $F_{-} P L U S \_T I M E O U T$ strategy.

For each of the three parameters, several values were tested, maintaining the other parameters with the base values. This led to the variations enumerated in Table I.

\begin{tabular}{lll} 
Timeout & Strategy & F \\
\hline 200 & F_PLUS_TIMEOUT & 0.8 \\
400 & F_PLUS_TIMEOUT & 0.8 \\
600 & F_PLUS_TIMEOUT & 0.8 \\
400 & ONLY_TIMEOUT & $0.8^{a}$ \\
400 & TIMEOUT_PLUS_F & 0.8 \\
$400^{b}$ & ONLY_F & 0.8 \\
400 & F_PLUS_TIMEOUT & 0.5 \\
400 & F_PLUS_TIMEOUT & 0.9 \\
& TABLE I &
\end{tabular}

CONFIGURATIONS FOR THE NEEM EXTREMA IMPLEMENTATION.

\footnotetext{
${ }^{a}$ The ONLY_TIMEOUT strategy does not take $F$ into account.

${ }^{b}$ The $O N L Y \_F$ strategy does not take Timeout into account.
}

For each configuration, 100 runs were executed and the log files were analyzed to determine the number of rounds, and the total time until convergence of the Minimums vector.

\section{Diameter Estimation}

1) Relative Error: In order to estimate the expected accuracy of the diameter estimation technique, we simulated the method using three different network topologies (random networks, preferential attachment networks and random geometric 2D networks) and sizes (50, 500 and 5000 nodes networks).

For each topology and size, 150 graphs were generated. For random and preferential attachment graphs, average degrees of 5, 10 and 15 were used. For each, 50 graphs were created. For 2D graphs, 1.3, 1.6 and 1.9 relative radius were used and 50 graphs were created for each.

For each graph, three different values of $K$ were simulated, 50 times each.

For a graph of size $N$, the simulation consisted of:

1) Generating $K$ random exponentially distributed values coded according to [4] for each node in the graph;

2) Calculating the $K$ global minimums across the graph;

3) Determining the nodes that generated each of the global minimums;

4) For each global minimum, calculating the shortest paths from all nodes to the nodes that generated the minimum and taking the minimum shortest path length;

5) Calculating the estimated diameter as the maximum of the previous values across all nodes.

For each graph, the diameter, radius and the eccentricities of all nodes were also calculated and recorded. This allowed to calculate the error in the diameter estimation and percentage of nodes in the periphery of the graph.

2) Rounds to converge: Convergence of the Hops vector cannot be determined by examining the log files, in normal circumstances. This happens because the overlay topology changes over time (NeEM "shuffles" peers periodically).

In order to get a rough idea of how long it might take for the Hops vector to converge in a static topology, a different test was performed. Before starting the Extrema protocol, all nodes were configured to disable shuffling, thus freezing the current overlay topology. A snapshot of the topology was then taken and recorded in a graph file for posterior analysis.

Two configurations were tested: one in which rounds are fast, because only half the neighbours are required in order to advance; and one in which rounds are slow, because nodes wait for every neighbour until a timeout and then proceed after a fraction of neighbours have responded. This corresponded to the following parameters:

- Fast rounds: TimeOut $=400$, Strategy = F_PLUS_TIMEOUT, $F=0.5$.

- Slow rounds: TimeOut $=400$, Strategy = TIMEOUT_PLUS_F, F $=0.8$.

For each value of $K \in\{10,100\}, 100$ runs were executed and recorded.

The log files were analyzed (specifically the Hops vector) to determine the error in the diameter estimation in the rounds after convergence of the Minimums vector. The overlay graph file served as reference for the true diameter of the network.

\section{Message Optimization}

In order to evaluate the message size improvements presented in Section IV-B, the implementation of Extrema in 
NeEM was extended in order to log, in each round, the number of values in the Minimums vector that needed to be sent to each neighbour, based on the comparison of the current Minimums vector and the last vector received from each neighbour. The same was done for the Hops vector.

The analysis was done considering two cases separately: messages without the Hops vector (only size estimation would be performed); and messages with the Hops vector (both size and diameter estimation would be performed).

The message size improvement procedure for the message with the Hops vector is analogous to the one presented in Section IV-B.

Five hundred runs were executed with parameters: Timeout $=400, F=0.5$, Strategy $=$ F_PLUS_TIMEOUT and $K=10$. Only networks with 65 nodes were tested.

\section{RESULTS AND DISCUSSION}

\section{A. Timeout and $F$}

The average number of rounds until convergence for the different combinations is shown in Figure 2.

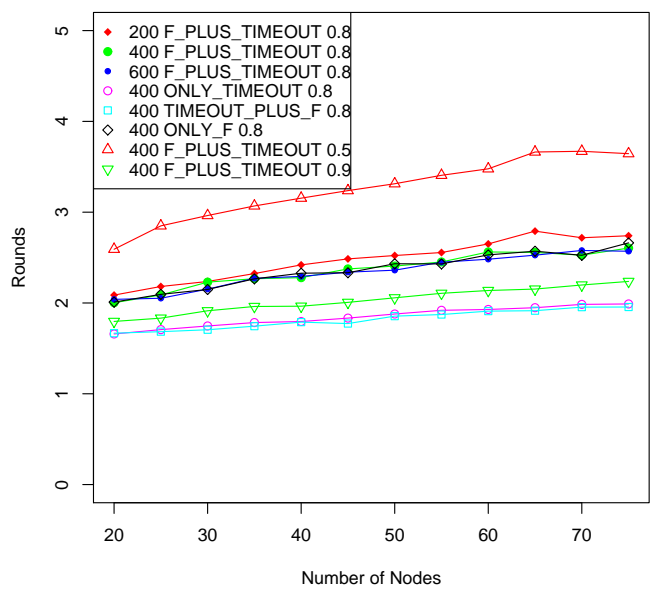

Fig. 2. Average number of rounds ellapsed until convergence.

The plots on Figure 2 indicates, from the number of rounds perspective, that:

- Trying to receive all possible messages before advancing round produces the best results. This is what happens in the ONLY_TIMEOUT and the TIMEOUT_PLUS_F settings.

- Waiting for only half of the neighbours is the worst setting. Clearly, in that setting, the timeout is not being triggered, meaning that rounds are advancing with only half the messages received increasing the number of needed rounds.

- A timeout of 400 milliseconds seems to be enough for most nodes to communicate, since the ONLY_TIMEOUT setting is very similar to the
TIMEOUT_PLUS_F with $F=0.8$. This means that at least $80 \%$ of the neighbours communicate within the 400 milliseconds interval.

Figure 3 shows the average time a node needs to achieve convergence. It indicates, from the total time perspective, that:

- Slower rounds, although implying less number of rounds, produce worst results. This can be seen from the top lines, which correspond to the better settings from the number of rounds point of view.

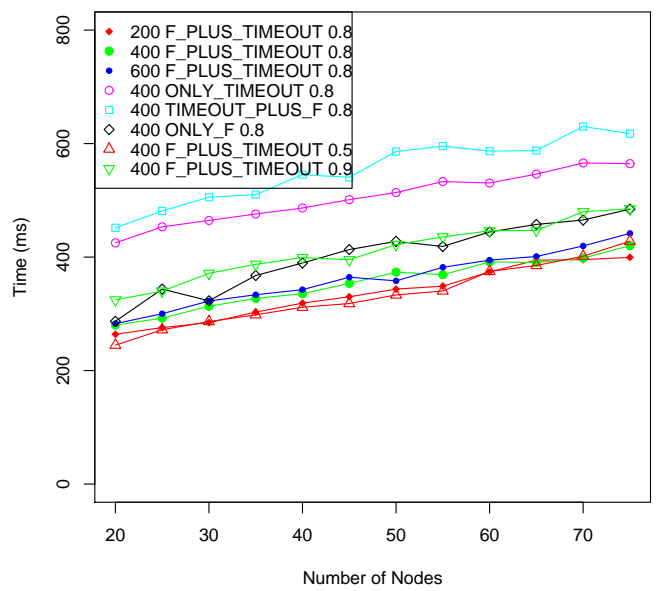

Fig. 3. Average time until convergence.

Comparing both Figures, its apparent that there is a tradeoff between time and communication (the number of rounds is an indirect measure of messages sent): faster convergence means higher communication cost.

1) No news Rounds: Although convergence of the Minimums vector can be determined by examining the log files, it cannot be determined by individual nodes. Nodes have to assume convergence after a predefined number of rounds have elapsed without changes in the Minimums vector.

In the previous tests, the maximum number of no news rounds $(\mathrm{NN})$ observed were the following:

$\begin{array}{llll}\text { Timeout } & \text { Strategy } & \text { F } & \text { NN } \\ 200 & \text { F_PLUS_TIMEOUT } & 0.8 & 5 \\ 400 & \text { F_PLUS_TIMEOUT } & 0.8 & 3 \\ 600 & \text { F_PLUS_TIMEOUT } & 0.8 & 4 \\ 400 & \text { ONLY_TIMEOUT } & 0.8 & 2 \\ 400 & \text { TIMEOUT_PLUS_F } & 0.8 & 2 \\ 400 & \text { ONLY_F } & 0.8 & 4 \\ 400 & \text { F_PLUS_TIMEOUT } & 0.5 & 7 \\ 400 & \text { F_PLUS_TIMEOUT } & 0.9 & 3\end{array}$

Although the values are fairly similar, it appears that faster rounds lead to a larger $\mathrm{NN}$ value than slower rounds.

\section{B. Diameter Estimation}

1) Relative Error: Table II summarizes the result of the simulation. 
Results indicate that the diameter is estimated with a maximum relative error of about $10 \%$, when $K=10$. However, increasing $K$ to 100 , reduces this error to less than $4 \%$.

From the absolute error perspective, random geometric 2D networks are clearly the worst type of network for the diameter estimation technique. In these networks, the number of nodes in the periphery is almost always very low. This leads to the highest average absolute error, from all types of networks. However, since geometric 2D networks also have the highest diameter, the relative error ends up being in the same order of magnitude (or very close) for all network types.

2) Rounds to Converge: Figure 4 shows the average absolute error in the diameter estimation as a function of the number of rounds after convergence of the Minimums vector, for different values of $K$.

In both configurations, it is apparent that the Hops vector (used to estimate the diameter) takes more time to converge than the Minimums vector. This happens because some shorter paths are slower than longer paths. So it may happen that a hop count is substituted by a lower count that came from a shorter, slower path. However, the same node may already have the final Minimums vector. Slower rounds, in which nodes wait a long time before advancing, tend to mask this effect since nodes on fast paths will still have to wait a long time before advancing.

\section{Message Optimization}

Table III summarizes the results and shows the average message size reduction in a run of the algorithm, per node, for different values of $\mathrm{T}$, with and without the Hops vector included in the message.

As expected, the overall message size reduction increases as $T$ increases. This happens because, near the final rounds of

\begin{tabular}{|c|c|c|c|c|}
\hline $\begin{array}{l}\text { Network } \\
\text { Type }\end{array}$ & $\begin{array}{l}\text { Network } \\
\text { Size }\end{array}$ & $\begin{array}{l}\text { Average } \\
\text { Diameter }\end{array}$ & $\begin{array}{l}\text { Average } \\
\text { Absolute } \\
\text { Error }\end{array}$ & $\begin{array}{r}\text { Average } \\
\text { Relative } \\
\text { Error }\end{array}$ \\
\hline \multicolumn{5}{|c|}{$K=10$} \\
\hline $2 \mathrm{~d}$ & 50 & 9.9 & 0.5997 & $6.03 \%$ \\
\hline Attach & 50 & 3.0 & 0.1116 & $3.68 \%$ \\
\hline Random & 50 & 2.4 & 0.0340 & $1.05 \%$ \\
\hline $2 \mathrm{~d}$ & 500 & 35.1 & 3.2631 & $9.43 \%$ \\
\hline Attach & 500 & 3.7 & 0.3396 & $8.14 \%$ \\
\hline Random & 500 & 4.0 & 0.4376 & $10.10 \%$ \\
\hline \multicolumn{5}{|c|}{$K=100$} \\
\hline $2 d$ & 50 & 9.9 & 0.0117 & $0.12 \%$ \\
\hline Attach & 50 & 3.0 & 0.0001 & 0\% \\
\hline Random & 50 & 2.4 & 0.0017 & $0.06 \%$ \\
\hline $2 \mathrm{~d}$ & 500 & 35.1 & 0.6669 & $2.00 \%$ \\
\hline Attach & 500 & 3.7 & 0.1588 & $3.73 \%$ \\
\hline Random & 500 & 4.0 & 0.1081 & $2.65 \%$ \\
\hline \multicolumn{5}{|c|}{$K=1000$} \\
\hline $2 \mathrm{~d}$ & 50 & 9.9 & 0 & $0 \%$ \\
\hline Attach & 50 & 3.0 & 0 & $0 \%$ \\
\hline Random & 50 & 2.4 & 0 & 0\% \\
\hline $2 \mathrm{~d}$ & 500 & 35.1 & 0.0116 & $0.03 \%$ \\
\hline Attach & 500 & 3.7 & 0.0048 & $0.11 \%$ \\
\hline Random & 500 & 4.0 & 0.0019 & $0.05 \%$ \\
\hline
\end{tabular}

Fast Rounds

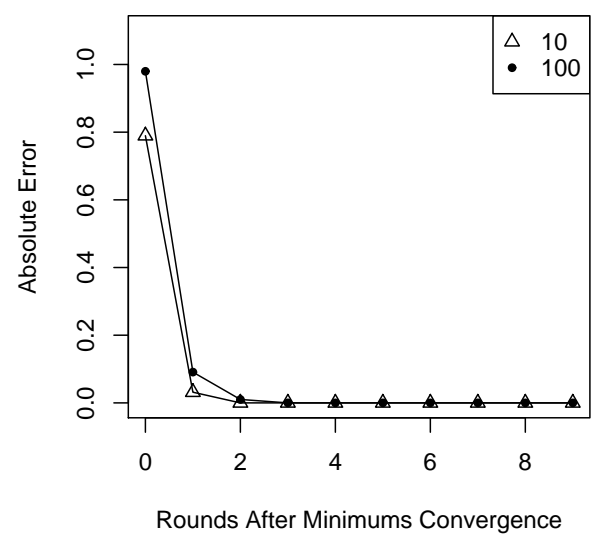

Slow Rounds

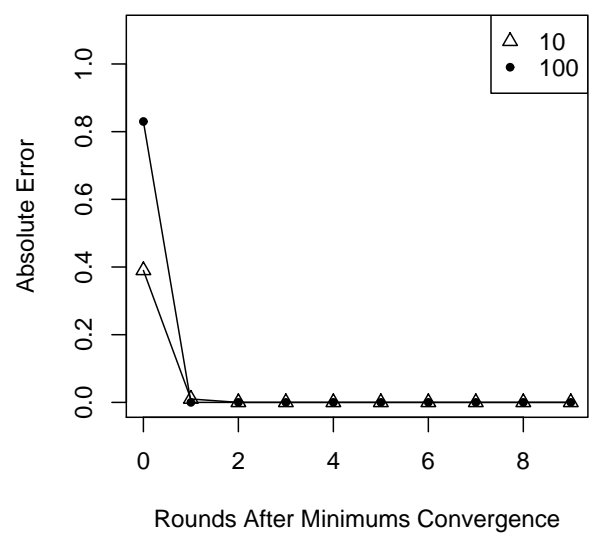

Fig. 4. Additional rounds needed to estimate the diameter.

\begin{tabular}{rrrr}
\multicolumn{4}{c}{ Without Hops vector } \\
\hline $\mathrm{T}$ & $\begin{array}{r}\text { Average } \\
\text { reduction }\end{array}$ & $\begin{array}{r}\text { Standard } \\
\text { deviation }\end{array}$ & $\begin{array}{r}\text { Average vector } \\
\text { length }\end{array}$ \\
0 & $3.37 \%$ & $1.38 \%$ & 7.94 \\
1 & $8.62 \%$ & $1.26 \%$ & 6.88 \\
2 & $13.91 \%$ & $1.03 \%$ & 5.94 \\
3 & $18.34 \%$ & $0.86 \%$ & 5.19 \\
4 & $21.85 \%$ & $0.75 \%$ & 4.61 \\
5 & $24.66 \%$ & $0.67 \%$ & 4.14 \\
6 & $26.95 \%$ & $0.62 \%$ & 3.77 \\
& & & \\
5 & & With Hops vector & \\
\hline $\mathrm{T}$ & Average & Standard & Average vector \\
& reduction & deviation & length \\
0 & $7.97 \%$ & $2.12 \%$ & 7.95 \\
1 & $15.63 \%$ & $1.95 \%$ & 6.92 \\
2 & $23.18 \%$ & $1.61 \%$ & 5.98 \\
3 & $29.44 \%$ & $1.34 \%$ & 5.24 \\
4 & $34.40 \%$ & $1.15 \%$ & 4.65 \\
5 & $38.38 \%$ & $1.02 \%$ & 4.18 \\
6 & $41.62 \%$ & $0.93 \%$ & 3.80 \\
& & & TABLE III
\end{tabular}

AVERAGE MESSAGE SIZE REDUCTION ACHIEVED USING THE IMPROVEMENTS PRESENTED IN SECTION IV-B. 
the algorithm, almost all nodes have the final vectors and their neighbours know this. This allows each node to send only a few values to each neighbour instead of the total $K$ values.

When considering the combined techniques for size and diameter estimation (message with Minimums and Hops vector), the reduction in message size is more pronounced than when considering only the size estimation (only Minimums vector). This happens because, in our implementation, each value in the Hops vector was coded using 8 bit, which in most cases would be too conservative a value. This leads to a greater reduction in size when using the improved message format.

The average vector length column in Table III shows the average number of values sent by each node to each neighbour ( $K$ was set to 10$)$. With three safe-guard rounds $(T=3)$ only about five values out of ten have to be sent, on average. For the two cases (only Minimums vector and Minimums plus Hops vector) the average vector length is very similar (which might contradict the difference in the values of the average reduction column, but that was explained in the previous paragraph).

\section{CONCLUSIONS}

This article presented a study on the behaviour of the Extrema Propagation technique on asynchronous scenarios, with different configuration settings. It also presented and evaluated a novel diameter estimation technique based on the Extrema Propagation technique.

When arbitrary delays and failures are allowed in the system model, the original Extrema Propagation algorithm has to be slightly modified. Instead of waiting for every neighbour, which could lead to deadlocks, each node should only wait for a fraction of its neighbours, wait until a timeout occurs, or a combination of the two.

Results show that there is a clear trade-off that must be considered. Fast rounds - rounds in which each node advances rapidly either because they wait for a small fraction of the neighbours or because a small timeout was configured - lead to a faster convergence of the Minimums vector and hence a faster estimation of the network size. However, fast rounds also lead to a larger number of rounds needed, which is an indirect measure of the number of messages exchanged. Slow rounds - in which each node waits for a large number of neighbours - lead to slower estimation but also to a smaller number of messages exchanged. The trade-off between communication cost and time must the considered.

Although the Extrema Propagation technique is already very efficient in terms of number of steps, since it come very close to the theoretical minimum of Diameter steps (with only a small constant increase due to the extra safe-guard rounds needed), it is still amenable to improvements in the size of the message that is used. The proposed improvement described in section IV-B shows that its possible to reduce the total communication cost using a simple approach. Even without considering additional safe-guard rounds, there is an average reduction of $3.4 \%$ in message size when considering only the basic Extrema Propagation technique and almost $8 \%$ when combining the diameter estimation technique. When aditional safe-guard rounds are used, the savings are even more substantial, rising to $18 \%$ and $29 \%$ for 3 aditional safe-guard rounds.

A diameter estimation technique based on the Extrema Propagation was presented and evaluated. The technique takes advantage of the generation of a fixed number of global minimums that act as a node sampling means to calculate the eccentricities of those nodes. If one of the "sampled" nodes is a peripheral node and enough rounds are allowed to pass, then the exact network diameter can be calculated. The accuracy can be adjusted by the parameter $K$, the number of minimums to generate. Results from simulations show that even with a very small number of minimums $(K=10)$, the relative error in the estimation is only about $10 \%$.

\section{AKNOWLEDGMENTS}

The authors would like to thank José Orlando Pereira and Nuno Carvalho for their help in the NeEM integration and testing. This work has been partially supported by FCT and POCI 2010, co-funded by the Portuguese Government and European Union in the FEDER Program.

\section{REFERENCES}

[1] P. Eugster, S. Handurukande, R. Guerraoui, A. Kermarrec, and P. Kouznetsov, "Lightweight probabilistic broadcast," 2001. [Online]. Available: citeseer.ist.psu.edu/eugster01lightweight.html

[2] A. J. Ganesh, A.-M. Kermarrec, and L. Massoulie, "Peer-to-peer membership management for gossip-based protocols," IEEE Transactions on Computers, vol. 52, no. 2, pp. 139-149, 2003.

[3] C. Baquero, P. Almeida, and R. Menezes, "Extrema propagation: Fast distributed estimation of sums and network sizes," Universidade do Minho, Tech. Rep., May 2006.

[4] C. Baquero, P. S. Almeida, and R. Menezes, "Fast estimation of aggregates in unstructured networks," in International Conference on Autonomic and Autonomous Systems (ICAS), Valencia, Spain, 20/04/2009. 25/04/2009. http://www.computer.org: IEEE Computer Society, april 2009.

[5] "Neem - network-friendly epidemic multicast," http://neem.sourceforge. net/index.html.

[6] D. Psaltoulis, D. Kostoulas, I. Gupta, K. Birman, and A. Demers, "Practical algorithms for size estimation in large and dynamic groups," University of Illinois, Urbana, Tech. Rep., 2004.

[7] D. Kempe, A. Dobra, and J. Gehrke, "Gossip-based computation of aggregate information," Foundations of Computer Science, 2003. Proceedings. 44th Annual IEEE Symposium on, pp. 482-491, October 2003.

[8] M. Jelasity, A. Montresor, and O. Babaoglu, "Gossip-based aggregation in large dynamic networks," ACM Trans. Comput. Syst., vol. 23, no. 1, pp. 219-252, 2005.

[9] J. Pereira, L. Rodrigues, M. J. Monteiro, R. Oliveira, and A.-M. Kermarrec, "Neem: Network-friendly epidemic multicast," in Proc. 22nd International Symposium on Reliable Distributed Systems, IEEE. Florence, Italy: IEEE Computer Society, October 2003.

[10] A. Vahdat, K. Yocum, K. Walsh, P. Mahadevan, D. Kostic, J. Chase, and D. Becker, "Scalability and accuracy in a large-scale network emulator," in Proceedings of 5th Symposium on Operating Systems Design and Implementation (OSDI), December 2002. [Online]. Available: http://www.cs.ucsd.edu/ vahdat/papers/modelnet.pdf

[11] D. Becker and K. Yocum, "Modelnet howto," Duke University., Tech. Rep., 2003, http://modelnet.ucsd.edu/howto.html. [Online]. Available: http://modelnet.ucsd.edu/howto.html 